\title{
Influences of spin accumulation on the intrinsic spin Hall effect in two-dimensional electron gases with Rashba spin-orbit coupling
}

\author{
Xiaohua Ma, ${ }^{1,2}$ Liangbin Hu, ${ }^{2,3}$ Ruibao Tao, ${ }^{1}$ and Shun-Qing Shen ${ }^{1,2}$ \\ ${ }^{1}$ State Key Laboratory of Applied Surface Physics and Department of Physics, Fudan University, Shanghai 200433, China \\ ${ }^{2}$ Department of Physics, The University of Hong Kong, Pokfulam Road, Hong Kong, China \\ ${ }^{3}$ Department of Physics, South China Normal University, Guangdong 510631, China
}

(Received 15 April 2004; published 29 November 2004)

\begin{abstract}
In a two-dimensional electron gas with Rashba spin-orbit coupling, the external electric field may cause a spin Hall current in the direction perpendicular to the electric field. This effect was called the intrinsic spin Hall effect. In this paper, we investigate the influences of spin accumulation on this intrinsic spin Hall effect. We show that due to the existence of boundaries in a real sample, the spin Hall current generated by the intrinsic spin Hall effect will cause spin accumulation near the edges of the sample, and in the presence of spin accumulation, the spin Hall conductivity will not have a universal value. The influences of spin accumulation on the intrinsic spin Hall effect in narrow strips of two-dimensional electron gases with Rashba spin-orbit coupling are investigated in detail.
\end{abstract}

DOI: 10.1103/PhysRevB.70.195343

PACS number(s): 72.10.-d, 72.15.Gd, 73.50.Jt

\section{INTRODUCTION}

Spintronics, which aims at the manipulation of the electron's spin degree of freedom in electronic devices, has become an emerging field of condensed matter physics for its potential application in information industry. ${ }^{1-3}$ Though originally many spintronic concepts involve ferromagnetic metals since spins in this kind of system behave collectively and hence are easy to be controlled, ${ }^{4-8}$ spintronics in semiconductors is more interesting because doped and heterojunction formation can be used to design some specific devices. It is anticipated that combining the advantages of semiconductors with the concepts of spintronics will yield fascinating new electronic devices and open the way to a new field of physics, i.e., semiconductor spintronics. However, at present many great challenges still remain in this exciting quest. Among them, an issue that is fundamentally important in semiconductor spintronics and has not yet been resolved is how to achieve efficient injections of spins into nonmagnetic semiconductors at room temperature. ${ }^{9-13}$ The usage of ferromagnetic metals as sources of spin injection is not practical because most of the spin polarizations will be lost at the interface between the metal and the semiconductor due to the large conductivity mismatch. ${ }^{9,10}$ Another possible approach is to use ferromagnetic semiconductors (such as $\mathrm{Ga}_{1-x} \mathrm{Mn}_{x} \mathrm{As}$ ) instead of ferromagnetic metals as sources of spin injection. In this approach, the problem of conductivity mismatch does not exist and hence efficient injections of spins into nonmagnetic semiconductors can truly be achieved. ${ }^{11-13}$ But for practical use at room temperatures, the Curie temperatures of ferromagnetic semiconductors are still too low. Thus, from both the experimental and theoretical points of view, more great efforts are still needed in order to achieve efficient injections of spins in nonmagnetic semiconductors at room temperatures. Recently, a surprising effect was predicted theoretically that an electric field may cause a quantum spin Hall current in the direction perpendicular to the electric field in conventional hole-doped semiconductors (such as $\mathrm{Si}, \mathrm{Ge}$, and $\mathrm{GaAs})^{14}$ or in two dimensional electron gases (2DEGs) with Rashba spin-orbit coupling. ${ }^{15}$ This intrinsic spin Hall effect might offer a new approach for achieving efficient injections of spins in nonmagnetic semiconductors and reveal a new avenue in the spintronics research.

In this paper, we study the influences of spin accumulation on the intrinsic spin Hall effect in 2DEGs with Rashba spin-orbit coupling. From the standpoint of spintronic applications, it is important to understand whether the spin Hall currents predicted in Refs. 14 and 15 are transport spin currents, i.e., whether they can be employed for transporting spins. An important feature of transport spin currents is that they will induce nonequilibrium spin accumulation at some specific locations, for example, at the boundaries of a sample or at the interfaces between two different materials. ${ }^{6-8}$ On the other hand, if spin accumulation was caused in a sample due to the flow of a spin current, the spin current will also be changed significantly by the spin accumulation. ${ }^{6-8}$ So in a full treatment of the intrinsic spin Hall effect, the interplay between the spin Hall current and the spin accumulation must be taken into account. A detailed theoretical investigation of the influences of spin accumulation on the intrinsic spin Hall effect in hole-doped semiconductors was presented in Ref. 16. In the present paper, we will use a similar method as was applied in Ref. 16 to investigate the influences of spin accumulation on the intrinsic spin Hall effect in 2DEGs with Rashba spin-orbit coupling. We will show that in contrast with what was found in Refs. 15, 17, and 18, in the presence of spin accumulation, the spin Hall conductivity in the intrinsic spin Hall effect in a 2DEG with Rashba spin-orbit coupling does not have a universal value, and in order to calculate correctly the spin Hall current and the spin Hall conductivity in a real sample with boundaries, the influences of spin accumulation need to be taken into account. The paper will be organized as follows: In Sec. II, we will first present a brief introduction to the intrinsic spin Hall effect in a 2 DEG in the absence of spin accumulation and impurity 
scattering. In Sec. III, the influences of spin accumulation and impurity scattering will be taken into account. In Sec. IV, by use of the formulas derived in Sec. III, the influences of spin accumulation on the intrinsic spin Hall effect in a narrow strip of a 2DEG will be discussed in detail.

\section{INTRINSIC SPIN HALL EFFECT IN THE ABSENCE OF SPIN ACCUMULATION AND IMPURITY SCATTERING}

In this paper, we will use a slightly different method from what was applied in Refs. 15 and 17 to discuss the intrinsic spin Hall effect in 2DEGs with Rashba spin-orbit coupling. The merit of this method is that the influences of spin accumulation can be easily included. For clarity, in this section we first present a brief introduction to the intrinsic spin Hall effect in the absence of spin accumulation and impurity scattering. We will show that our method will recover the same results as was obtained in Refs. 15 and 17 if spin accumulation and impurity scattering are neglected.

In the momentum representation, the single-particle Hamiltonian for a 2DEG with Rashba spin-orbit coupling reads ${ }^{19}$ as

$$
\hat{H}_{0}=\frac{\hbar^{2} k^{2}}{2 m}-\alpha\left(\mathbf{e}_{z} \times \mathbf{k}\right) \cdot \sigma=\frac{\hbar^{2} k^{2}}{2 m}-\alpha k\left(\cos \theta \sigma_{y}-\sin \theta \sigma_{x}\right),
$$

where $\mathbf{e}_{z}$ is the unit vector perpendicular to the twodimensional plane, $\mathbf{k}=(k \cos \theta, k \sin \theta)$ is the momentum of an electron, $\sigma=\left(\sigma_{x}, \sigma_{y}, \sigma_{z}\right)$ the Pauli matrices, and $\alpha$ the Rashba spin-orbit coupling constant. The Hamiltonian (1) can be diagonalized exactly. The eigenenergies are given by

$$
\epsilon_{\mathbf{k} \lambda}=\frac{\hbar^{2} k^{2}}{2 m}-\lambda \alpha k \quad(\lambda= \pm 1)
$$

and the corresponding spinor eigenstates are defined by

$$
|\mathbf{k} \lambda\rangle=\frac{1}{\sqrt{2}}\left(\begin{array}{c}
-i \lambda e^{-i \theta} \\
1
\end{array}\right) \text {. }
$$

The expectation value of the spin of an electron which is in the spinor eigenstate $|\mathbf{k} \lambda\rangle$ will be given by

$$
\mathbf{S}_{\lambda}^{(0)}(\mathbf{k})=\frac{\hbar}{2}\langle\mathbf{k} \lambda|\sigma| \mathbf{k} \lambda\rangle=S_{\lambda, x}^{(0)}(\mathbf{k}) \mathbf{e}_{x}+S_{\lambda, y}^{(0)}(\mathbf{k}) \mathbf{e}_{y},
$$

where $\mathbf{e}_{x}$ and $\mathbf{e}_{y}$ are the unit vectors along the $x$ and $y$ axes in the plane, respectively, and $S_{\lambda, x}^{(0)}(\mathbf{k})$ and $S_{\lambda, y}^{(0)}(\mathbf{k})$ are the $x$ and $y$ components of the spin, which are given by

$$
\begin{gathered}
S_{\lambda, x}^{(0)}(\mathbf{k})=\frac{\hbar}{2}\left\langle\mathbf{k} \lambda\left|\sigma_{x}\right| \mathbf{k} \lambda\right\rangle=\lambda \frac{\hbar k_{y}}{2 k}, \\
S_{\lambda, y}^{(0)}(\mathbf{k})=\frac{\hbar}{2}\left\langle\mathbf{k} \lambda\left|\sigma_{y}\right| \mathbf{k} \lambda\right\rangle=-\lambda \frac{\hbar k_{x}}{2 k} .
\end{gathered}
$$

Equations (4)-(6) show that the eigenstates of the Hamiltonian (1) are spin polarized in the in-plane directions, and the spin-polarization directions depend on the momentum $\mathbf{k}$.
When an external electric field is applied in the $x$ direction, the total Hamiltonian of the system will be given by $\hat{H}=\hat{H}_{0}+e \mathbf{E} \cdot \mathbf{x}$, where $\mathbf{E}=E_{x} \mathbf{e}_{x}$ is the external electric field and $-e$ the charge of the electron. The equation of motion for the electron's position and spin degrees of freedom under the action of the external electric field can be obtained directly from the Heisenberg equation, and the results read as

$$
\begin{gathered}
\frac{d \mathbf{k}}{d t}=\frac{e \mathbf{E}}{\hbar}, \\
\frac{d \mathbf{S}}{d t}=\mathbf{h}_{\mathrm{eff}} \times \mathbf{S},
\end{gathered}
$$

where $\mathbf{h}_{\text {eff }}=(2 \alpha / \hbar)\left(k_{y} \mathbf{e}_{x}-k_{x} \mathbf{e}_{y}\right)$ is an effective magnetic field felt by an electron with momentum $\mathbf{k}$ due to the Rashba spin-orbit coupling. The time variation of the electron spin which is initially in the spinor eigenstate $|\mathbf{k} \lambda\rangle$ [i.e., $\mathbf{S}(t=0)$ $=(\hbar / 2)\langle\mathbf{k} \lambda|\hat{\sigma}| \mathbf{k} \lambda\rangle]$ can be obtained by solving Eqs. (7) and (8) simultaneously. In this paper we will consider only the linear response of the transport property to the electric field. In the linear response regime, Eqs. (7) and (8) can be integrated analytically by use of the same method of Ref. 15, and one can find that under the action of the external electric field, the spin of an electron with momentum $\mathbf{k}$ and initially in the spinor eigenstate $|\mathbf{k} \lambda\rangle$ will become

$$
\begin{gathered}
S_{\lambda, x}(\mathbf{k}) \simeq S_{\lambda, x}^{(0)}(\mathbf{k}), \\
S_{\lambda, y}(\mathbf{k}) \simeq S_{\lambda, y}^{(0)}(\mathbf{k}), \\
S_{\lambda, z}(\mathbf{k}) \simeq-\lambda \frac{e \hbar k_{y}}{4 \alpha k^{3}} E_{x},
\end{gathered}
$$

where $S_{\lambda, i}(\mathbf{k})$ is the $i$ th component of the spin. Equations (9)-(11) show that an applied electric field in the $x$ direction will cause the spin to tilt in the perpendicular direction by an amount proportional to $k_{y}$. Due to this fact, the application of an external electric field in the $x$ direction will induce a spin Hall current in the $y$ direction with spin parallel to the $z$ direction. The spin Hall current can be calculated by the following formula:

$$
J_{y}^{S_{z}}=\sum_{\lambda} \int \frac{d^{2} \mathbf{k}}{(2 \pi)^{2}} S_{\lambda, z}(\mathbf{k})\left(\hbar k_{y} / m\right) f_{\lambda}(\mathbf{k}),
$$

where $f_{\lambda}(\mathbf{k})$ is the probability distribution function of conduction electrons. If spin accumulation and electron-impurity scattering can be neglected, $f_{\lambda}(\mathbf{k})$ can be given simply by the Fermi-Dirac equilibrium distribution function, i.e., $f_{\lambda}(\mathbf{k})=f^{0}\left(\epsilon_{\mathbf{k} \lambda}\right) \equiv\left[\exp \left[\beta\left(\epsilon_{\mathbf{k} \lambda}-\epsilon_{F}\right)\right]+1\right]^{-1}$, where $\beta=1 / k_{B} T$ and $\epsilon_{F}$ is the Fermi energy. Then one can find that in the usual case where both spin-orbit split bands are occupied, the spin Hall current $J_{y}^{S_{z}}$ and the spin Hall conductivity $\sigma_{s}$ will be given by ${ }^{15}$ 


$$
J_{y}^{S_{z}}=\sigma_{s} E_{x}, \quad \sigma_{s}=\frac{e}{8 \pi} .
$$

Equation (13) is the central result of Ref. 15, which was also obtained by several other groups with different theoretical approaches. ${ }^{17}$ Equation (13) shows that as long as both spinorbit split bands are occupied, the spin Hall conductivity will have a universal value, independent of both the Rashba spinorbit coupling strength and of the density of conduction electrons. Though Eq. (13) was obtained in the clean limit, recent numerical simulation shows that it is not suppressed completely in the presence of weak disorder, providing that the sample size exceeds the localization length. ${ }^{18}$ It is also important to note that unlike the similar effect conceived by Hirsch, ${ }^{20}$ which is caused by spin-orbit dependent anisotropic scattering from impurities and will vanish in the weak scattering limit, ${ }^{20-22}$ the spin Hall effect described by Eq. (13) has a quantum nature and is purely intrinsic, i.e., it does not rely on any anisotropic scattering from impurities. Of course, it should be pointed out that though the mechanism of the intrinsic spin Hall effect described above does not involve impurity scattering, it does not mean that impurity scattering has no significant influences on the effect. The reason is that in a real sample, due to the existence of boundaries, nonequilibrium spin accumulation will be caused inevitably near the edges of the sample when the spin Hall current circulates in it, and in the presence of nonequilibrium spin accumulation, spin diffusion will be induced by electron-impurity scattering and, hence, the spin Hall current may also be changed significantly from what was given by Eq. (13). Thus in order to calculate correctly the spin Hall current and the spin Hall conductivity in a real sample with boundaries, the influences of spin accumulation and electronimpurity scattering need to be considered.

\section{INFLUENCES OF SPIN ACCUMULATION AND IMPURITY SCATTERING}

In the presence of spin accumulation and electronimpurity scattering, the distribution function of conduction electrons can no longer be given simply by the Fermi-Dirac equilibrium distribution function but may be derived by solving the Boltzmann transport equation. In a steady (but nonequilibrium) state, the Boltzmann equation reads as

$$
\begin{gathered}
\mathbf{V}_{\lambda}(\mathbf{k}) \cdot \nabla f_{\lambda}(\mathbf{r}, \mathbf{k})-e \mathbf{E}_{\text {ext }} \cdot \mathbf{V}_{\lambda}(\mathbf{k}) \frac{\partial f_{\lambda}(\mathbf{r}, \mathbf{k})}{\partial \epsilon_{\lambda}(k)} \\
=-\left[\left(\frac{\partial f_{\lambda}}{\partial t}\right)_{\text {coll. }}^{(\lambda \rightarrow \lambda)}+\left(\frac{\partial f_{\lambda}}{\partial t}\right)_{\text {coll. }}^{(\lambda \rightarrow \bar{\lambda})}\right],
\end{gathered}
$$

where $\mathbf{V}_{\lambda}(\mathbf{k})=\nabla_{\mathbf{k}} \epsilon_{\mathbf{k} \lambda} / \hbar$ is the velocity of conduction electrons, $\mathbf{E}_{\text {ext }}=E_{x} \mathbf{e}_{x}$ is the external electric field applied in the $x$ direction, and $f_{\lambda}(\mathbf{r}, \mathbf{k})$ is the distribution function. The collision term $\left(\partial f_{\lambda} / \partial t\right)_{\text {coll. }}^{\left(\lambda \rightarrow \lambda^{\prime}\right)}$ describes the changes of the distribution function due to the intra-band $\left(\lambda^{\prime}=\lambda\right)$ and/or interband $\left(\lambda^{\prime}=\bar{\lambda}\right)$ electron-impurity scattering, which is given by

$$
\begin{aligned}
\left(\frac{\partial f_{\lambda}}{\partial t}\right)_{\text {coll }}^{\left(\lambda \rightarrow \lambda^{\prime}\right)}= & -\int \frac{d^{2} k^{\prime}}{(2 \pi)^{2}} \omega_{\lambda, \lambda^{\prime}}\left(\mathbf{k}, \mathbf{k}^{\prime}\right) \delta\left(\epsilon_{\lambda}(\mathbf{k})-\epsilon_{\lambda^{\prime}}\left(\mathbf{k}^{\prime}\right)\right) \\
& \times\left[f_{\lambda}(\mathbf{r}, \mathbf{k})-f_{\lambda^{\prime}}\left(\mathbf{r}, \mathbf{k}^{\prime}\right)\right]
\end{aligned}
$$

where $\omega_{\lambda, \lambda^{\prime}}\left(\mathbf{k}, \mathbf{k}^{\prime}\right)$ is the rates of an electron to be scattered from the state $|\mathbf{k} \lambda\rangle$ into the state $\left|\mathbf{k}^{\prime} \lambda^{\prime}\right\rangle$ by impurity scattering.

The Boltzmann equation (14) can be solved by the relaxation time approximation method. Within the relaxation time approximation and in the linear response regime, the system can be considered only slightly deviated from the equilibrium state, thus the total distribution $f_{\lambda}(\mathbf{r}, \mathbf{k})$ can be expressed as the sum of the equilibrium distribution function $f^{0}\left(\epsilon_{\mathbf{k} \lambda}\right)$ and the nonequilibrium ones as the following:

$$
\begin{aligned}
f_{\lambda}(\mathbf{r}, \mathbf{k})= & f^{0}\left(\epsilon_{\mathbf{k} \lambda}\right)-e \mu^{\lambda}(\mathbf{r}) \frac{\partial f^{0}\left(\epsilon_{\mathbf{k} \lambda}\right)}{\partial \epsilon_{\mathbf{k} \lambda}} \\
& +e \tau_{\lambda}(\mathbf{k}) \mathbf{E}^{\lambda}(\mathbf{r}) \cdot \mathbf{V}_{\lambda}(\mathbf{k}) \frac{\partial f^{0}\left(\varepsilon_{\mathbf{k} \lambda}\right)}{\partial \varepsilon_{\mathbf{k} \lambda}}
\end{aligned}
$$

Here the second term denotes the change of the distribution function due to the occurrence of nonequilibrium spin accumulation in the sample, with $-e \mu^{\lambda}(\mathbf{r})(\lambda= \pm)$ denoting the band- and position-dependent shifts of the Fermi level in the nonequilibrium state, which characterize the imbalance of the filling of conduction electrons in the two spin-orbit split bands in the presence of nonequilibrium spin accumulation. The third term in Eq. (16) denotes the changes of the distribution function due to the movement of conduction electrons under the action of an effective electric field $\mathbf{E}^{\lambda}(\mathbf{r})$, with $\tau_{\lambda}(\mathbf{k})$ denoting the total relaxation time of conduction electrons with momentum $\mathbf{k}$, which is determined by the electron-impurity scattering. Because the occurrence of nonequilibrium spin accumulation will cause spin diffusion in the sample, the effective electric field $\mathbf{E}^{\lambda}(\mathbf{r})$ should be given by $\mathbf{E}^{\lambda}(\mathbf{r})=\mathbf{E}_{\text {ext }}-\nabla \mu^{\lambda}(\mathbf{r})$, i.e., in addition to the external electric field $\mathbf{E}_{\text {ext }}$, conduction electrons will also feel an effective field given by the gradients of the band- and positiondependent shifts of the Fermi level. ${ }^{6-8}$ Substituting Eq. (16) into Eqs. (14) and (15), the left-hand side of the Boltzmann equation becomes

$$
\begin{aligned}
L h s= & -e \frac{\partial f^{0}\left(\epsilon_{\mathbf{k} \lambda}\right)}{\partial \epsilon_{\mathbf{k} \lambda}}\left\{\mathbf{E}^{\lambda}(\mathbf{r}) \cdot \mathbf{V}_{\lambda}(\mathbf{k})\right. \\
& \left.-\tau_{\lambda}(\mathbf{k}) \mathbf{V}_{\lambda}(\mathbf{k}) \cdot \nabla\left[\mathbf{E}^{\lambda}(\mathbf{r}) \cdot \mathbf{V}_{\lambda}(\mathbf{k})\right]\right\},
\end{aligned}
$$

and the right-hand side of the Boltzmann equation becomes

$$
\begin{aligned}
R h s= & -e \frac{\partial f^{0}\left(\epsilon_{\mathbf{k} \lambda}\right)}{\partial \epsilon_{\mathbf{k} \lambda}}\left\{\left[\frac{\tau_{\lambda}(\mathbf{k})}{\tau_{\lambda}^{\uparrow \uparrow}(\mathbf{k})}+\frac{\tau_{\lambda}(\mathbf{k})}{\tau_{\lambda}^{\uparrow \downarrow}(\mathbf{k})}\right]\left[\mathbf{E}^{\lambda}(\mathbf{r}) \cdot \mathbf{V}_{\lambda}(\mathbf{k})\right]\right. \\
& \left.-\frac{\mu^{\lambda}(\mathbf{r})-\mu^{-\lambda}(\mathbf{r})}{\tau_{\lambda}^{\uparrow \downarrow}(\mathbf{k})}\right\},
\end{aligned}
$$

where $\tau_{\lambda}^{\uparrow}(\mathbf{k})$ and $\tau_{\lambda}^{\uparrow \downarrow}(\mathbf{k})$ are the intra-band and inter-band transition relaxation times, respectively, which are defined by 


$$
\begin{gathered}
\tau_{\lambda}^{\uparrow \uparrow}(\mathbf{k})=\left[\int \frac{d^{2} k^{\prime}}{(2 \pi)^{2}} \omega_{\lambda, \lambda}\left(\mathbf{k}, \mathbf{k}^{\prime}\right) \delta\left(\epsilon_{\lambda}(\mathbf{k})-\epsilon_{\lambda}\left(\mathbf{k}^{\prime}\right)\right)\right]^{-1} ; \\
\tau_{\lambda}^{\uparrow \downarrow}(\mathbf{k})=\left[\int \frac{d^{2} k^{\prime}}{(2 \pi)^{2}} \omega_{\lambda,-\lambda}\left(\mathbf{k}, \mathbf{k}^{\prime}\right) \delta\left(\epsilon_{\lambda}(\mathbf{k})-\epsilon_{-\lambda}\left(\mathbf{k}^{\prime}\right)\right)\right]^{-1} .
\end{gathered}
$$

By integrating both the left-hand side and the right-hand side of the Boltzmann equation, one can find that the total relaxation time $\tau_{\lambda}$ of conduction electrons should be given by

$$
\tau_{\lambda}(\mathbf{k})=\left[\frac{1}{\tau_{\lambda}^{\uparrow \uparrow}(\mathbf{k})}+\frac{1}{\tau_{\lambda}^{\uparrow \downarrow}(\mathbf{k})}\right]^{-1},
$$

and the band- and position-dependent shifts of the Fermi level satisfy the following equation:

$$
\nabla^{2} \mu^{\lambda}(\mathbf{r})=\frac{\mu^{\lambda}(\mathbf{r})-\mu^{-\lambda}(\mathbf{r})}{D_{\lambda}^{2}},
$$

where $D_{\lambda} \equiv\left[\left(V_{F}^{\lambda}\right)^{2} \tau_{F} \tau_{F}^{\uparrow \downarrow}\right]^{1 / 2}$, with $V_{F}^{\lambda}$ denoting the banddependent Fermi velocity and $\tau_{F}^{\uparrow \downarrow}$ the inter-band-transition relaxation time and $\tau_{F}$ the total relaxation time of conduction electrons at the Fermi level, respectively. (For simplicity, we assume that $\tau_{F}$ and $\tau_{F}^{\uparrow} \downarrow$ are band independent.) From Eq. (22), one can see that the relative shifts of the Fermi level in the two spin-orbit split bands, given by $\mu^{+}(\mathbf{r})-\mu^{-}(\mathbf{r})$, satisfy the following diffusion equation:

$$
\nabla^{2}\left[\mu^{+}(\mathbf{r})-\mu^{-}(\mathbf{r})\right]=\frac{\mu^{+}(\mathbf{r})-\mu^{-}(\mathbf{r})}{D^{2}},
$$

where $D$ is the spin-diffusion length, defined by

$$
D=\left[\frac{1}{D_{+}^{2}}+\frac{1}{D_{-}^{2}}\right]^{-1 / 2} .
$$

In addition to Eqs. (22) and (23), the band-dependent shifts of the Fermi level should also satisfy the charge neutrality condition, which requires that the net changes of the charge density due to the band-dependent shifts of the Fermi level, given by $\delta \rho(\mathbf{r})=e \Sigma_{\lambda} \int\left[d^{2} k /(2 \pi)^{2}\right]\left[f_{\lambda}(\mathbf{r}, \mathbf{k})-f_{\lambda}^{0}\left(\epsilon_{\mathbf{k} \lambda}\right)\right]$, should be zero. This requirement arises from the fact that according to the symmetry of the Hamiltonian (1), in the direction perpendicular to the external electric field, no charge Hall current will be generated, so the occurrence of spin accumulation due to the flow of the spin Hall current does not result in charge accumulation. Due to this requirement, one can show that in addition to Eqs. (22) and (23), the band-dependent shifts of the Fermi level should also satisfy the following equation:

$$
\sum_{\lambda= \pm} k_{\lambda F} \mu^{\lambda}(\mathbf{r})=0
$$

where $k_{\lambda F}$ is the band-dependent wave number at the Fermi level. After the band-dependent shifts of the Fermi level are determined, the spin Hall current can be obtained by inserting Eq. (11) and Eq. (16) into Eq. (12); then one can find that in the usual case where both spin-orbit split bands are occupied, ${ }^{23}$ the spin Hall current and the spin Hall conductivity will be given by

$$
\begin{gathered}
J_{y}^{S_{z}}(\mathbf{r})=\sigma_{s}(\mathbf{r}) E_{x} \\
\sigma_{s}(\mathbf{r})=\frac{e}{8 \pi}\left(1-\frac{e\left[\mu^{+}(\mathbf{r})-\mu^{-}(\mathbf{r})\right]}{2 \alpha\left[\left(m \alpha / \hbar^{2}\right)^{2}+2 m \epsilon_{F} / \hbar^{2}\right]^{1 / 2}}\right) .
\end{gathered}
$$

Equations (26) and (27) show that the spin accumulation may have some significant influences on the intrinsic spin Hall effect in a 2DEG. First, in the presence of spin accumulation, the spin Hall conductivity will be a positiondependent quantity and does not have a universal value, i.e., it will depend on the Rashba spin-orbit coupling constant and on the density of conduction electrons. This is very different from what was shown in Eq. (13). Second, in the presence of spin accumulation, the spin Hall current may be decreased substantially from the corresponding value obtained in the absence of spin accumulation, and the decrease will be determined by $\mu^{+}(\mathbf{r})-\mu^{-}(\mathbf{r})$, i.e., proportional to the relative shifts of the Fermi level in the two spin-orbit split bands.

\section{RESULTS AND DISCUSSIONS}

Equations (23) and (25)-(27) constitute a set of selfconsistent equations, from which both the spin Hall current and the spin accumulation can be obtained. In this section, we apply these formulas to discuss the intrinsic spin Hall in a narrow strip of a $2 \mathrm{DEG}$ with Rashba spin-orbit coupling. Narrow strips are the usual geometry applied in the experimental measurement of the Hall effect, including the spin Hall effect. ${ }^{20-22}$ In the following we will assume that the longitudinal direction of the strip is along the $x$ axis and the transverse direction along the $y$ axis and the normal of the $2 \mathrm{D}$ plane along the $z$ axis, respectively, and an external electric field $E_{x}$ is applied in the longitudinal direction of the strip. According to Eqs. (26) and (27), in order to calculate the spin Hall current $J_{y}^{S_{z}}$ caused by the longitudinal external electric field $E_{x}$, one must first find out the band-dependent shifts $\mu^{+}(\mathbf{r})$ and $\mu^{-}(\mathbf{r})$ of the Fermi level. For simplicity, we assume that the length $L$ of the strip is much larger than its width $w$ so that spin diffusion in the longitudinal direction of the strip can be neglected. In such a case, only transverse spin accumulation needs to be considered, and $\mu^{\lambda}(\mathbf{r}), J_{y}^{S_{z}}(\mathbf{r})$, and $\sigma_{s}(\mathbf{r})$ will all depend only on the $y$ coordinates. From Eq. (23), $\mu^{+}(y)-\mu^{-}(y)$ can be expressed as

$$
\mu^{\lambda}(y)-\mu^{-\lambda}(y)=A e^{y / D_{1}}+B e^{-y / D_{1}},
$$

where $A$ and $B$ are two constant coefficients that need to be determined by appropriate boundary conditions. In this paper, we will consider the transverse-open-circuit boundary condition. In the transverse-open-circuit boundary condition, the spin Hall current at the two boundaries of the sample, which are assumed to be located at $y= \pm w / 2$, should be zero, i.e.,

$$
J_{y}^{S_{z}}\left(y= \pm \frac{w}{2}\right)=0 .
$$

Substituting Eq. (28) into Eqs. (26) and (27) and by use of the above boundary condition, the coefficients $A$ and $B$ can 


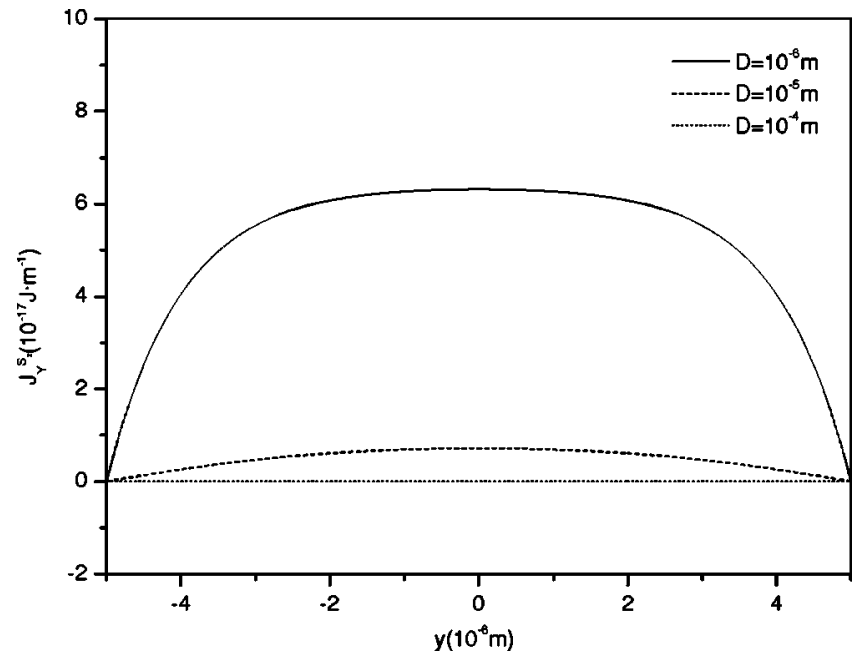

FIG. 1. An illustration of the transverse spatial distributions of the spin Hall currents in three distinct cases with different ratios of $D / w$. The parameters used are the sample width $w=10 \mu \mathrm{m}$; the spin diffusion length $D=1 \mu \mathrm{m}$ (the solid line), $10 \mu \mathrm{m}$ (the dashed line), and $100 \mu \mathrm{m}$ (the dotted line).

be determined. Then the band- and position-dependent shifts of the Fermi level in the strip can be obtained, and we get

$$
\begin{aligned}
& \mu^{+}(y)=\frac{M_{0} \cosh (y / D)}{2 \cosh (w / 2 D)}\left[1+\frac{D^{2}}{D_{0}^{2}}\right], \\
& \mu^{-}(y)=\frac{M_{0} \cosh (y / D)}{2 \cosh (w / 2 D)}\left[\frac{D^{2}}{D_{0}^{2}}-1\right],
\end{aligned}
$$

where $M_{0}$ and $D_{0}$ are defined by

$$
\begin{gathered}
M_{0}=\frac{2 \alpha}{e} \sqrt{\frac{m^{2} \alpha^{2}}{\hbar^{4}}+\frac{2 m \epsilon_{F}}{\hbar^{2}}}, \\
D_{0}=\left[\frac{1}{D_{+}^{2}}-\frac{1}{D_{-}^{2}}\right]^{-1 / 2} .
\end{gathered}
$$

Here $D_{\lambda}(\lambda= \pm)$ has been defined in Eq. (22). In obtaining Eqs. (30) and (31), the charge neutrality condition (25) was also used. After $\mu^{\lambda}(y)$ is determined, according to Eqs. (26) and (27), the spin Hall current and the spin Hall conductivity will also be obtained, and the results read as

$$
\begin{gathered}
J_{y}^{S_{z}}(y)=\sigma_{s}(y) E_{x}, \\
\sigma_{s}(y)=\frac{e}{8 \pi}\left[1-\frac{\cosh (y / D)}{\cosh (w / 2 D)}\right] .
\end{gathered}
$$

Equations (34) and (35) show that, due to the influences of spin accumulation, the spatial distribution of the spin Hall current in a sample will be highly inhomogeneous and the spin Hall conductivity is sensitively position dependent. The spin Hall current and the spin Hall conductivity and their spatial distributions will also have sensitive dependences on the spin diffusion length $D$ and the sample width $w$. This was shown in Fig. 1, where we have plotted the transverse spatial distributions of the spin Hall currents in three distinct cases with different ratios of $D / w$. From Fig. 1, one can see that if $w \ll D$, the spin Hall current will be negligibly small in the sample, i.e., $J_{y}^{S_{z}}(y) \simeq 0$ everywhere. On the other hand, if $w$ $\gg D$, the spin Hall conductivity will be approximately a constant at $|y| \ll w / 2$, i.e., $\sigma_{s}(y)=J_{y}^{S_{z}}(y) / E_{x} \simeq e / 8 \pi$ at $|y| \ll w / 2$, which is independent of both the Rashba spin-orbit coupling strength and of the density of conduction electrons. But $\sigma_{s}(y)$ will decrease to zero as $y \rightarrow \pm w / 2$.

The spin accumulation caused by the longitudinal electric field $E_{x}$ can be calculated through the following formula:

$$
\langle\mathbf{S}\rangle=\sum_{\lambda} \int \frac{d^{2} k}{(2 \pi)^{2}} \mathbf{S}_{\lambda}(\mathbf{k}) f_{\lambda}(\mathbf{r}, \mathbf{k}),
$$

where $\mathbf{S}_{\lambda}(\mathbf{k})$ has been given in Eqs. (9)-(11). By inserting Eq. (16) and Eqs. (9)-(11) into Eq. (36) and with the help of Eqs. (30) and (31), one can find that both the $y$ component of $\langle\mathbf{S}\rangle$ (the in-plane spin accumulation) and the $z$ component of $\langle\mathbf{S}\rangle$ (the perpendicular spin accumulation) are nonzero,

$$
\begin{gathered}
\left\langle S_{y}\right\rangle=\frac{e m \alpha \tau_{F} E_{x}}{4 \pi \hbar^{2}}, \\
\left\langle S_{z}\right\rangle=\frac{e E_{x}}{4 \pi}\left(\frac{m \tau_{F}}{\tau_{F}^{\uparrow \downarrow} \epsilon_{F}}\right)^{1 / 2}\left(1+\frac{\alpha^{2} m}{\hbar^{2} \epsilon_{F}}\right) \frac{\sinh (y / D)}{\cosh (w / 2 D)} .
\end{gathered}
$$

Equations (37) and (38) show that both the in-plane and the perpendicular spin accumulation are proportional to the longitudinal electric field $E_{x}$, but there are some significant differences between them. The in-plane spin accumulation is homogeneously distributed in the sample and independent of both the spin diffusion length $D$ and of the sample width $w$. However, the spatial distribution of the perpendicular spin accumulation is highly inhomogeneous and its magnitude depends sensitively on the spin diffusion length $D$ and on the sample width $w$. These differences arise from the fact that the in-plane and the perpendicular spin accumulation are caused by a very different mechanism. In fact, it was known long time ago that in a 2DEG with Rashba spin-orbit coupling, an applied in-plane electric field will induce a homogeneous in-plane spin accumulation polarized in the direction perpendicular to the electric field, ${ }^{24,25}$ but the in-plane spin accumulation has nothing to do with the intrinsic spin Hall effect. From the theoretical viewpoints, the in-plane spin accumulation is caused by the combined action of the spin-orbit coupling, absence of inversion symmetry, and the time-reversal symmetry-breaking in the electric field. ${ }^{24,25}$ Since the in-plane spin accumulation has been investigated in detail in previous literature and it has no relation with the intrinsic spin Hall effect, we will not discuss it again in the present paper. Unlike the in-plane spin accumulation, the perpendicular spin accumulation given by Eq. (38) is caused by the intrinsic spin Hall effect, so its spatial distribution is highly inhomogeneous and its magnitude depends sensitively on the spin diffusion length $D$ and on the sample width $w$. This was illustrated clearly in Fig. 2, where we have plotted the transverse spatial distributions of the perpendicular spin accumulation in three distinct cases with different ratios of $D / w$. From Fig. 2 and Eq. (38), one can see that the perpen- 


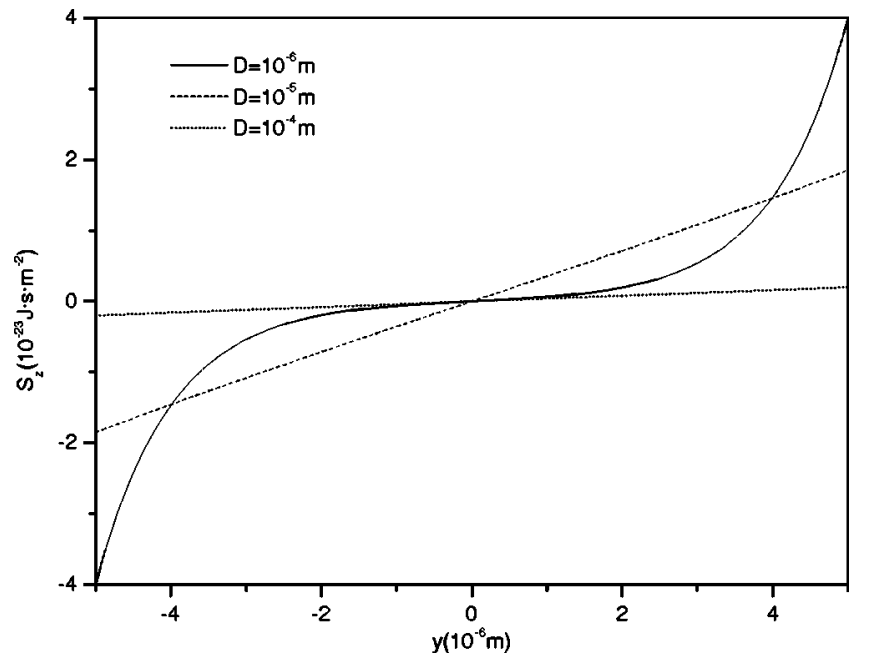

FIG. 2. The transverse spatial distributions of the perpendicular spin accumulation in three distinct cases with different ratios of $D / w$. The parameters used are the sample width $w=10 \mu \mathrm{m}$; the spin diffusion length $D=1 \mu \mathrm{m}$ (the solid line), $10 \mu \mathrm{m}$ (the dashed line), and $100 \mu \mathrm{m}$ (the dotted line); the external field $e E_{x}$ $=10 \mathrm{KeV} / \mathrm{m}$; the Rashba spin-orbit coupling constant $\alpha=1$ $\times 10^{-11} \mathrm{eV} \mathrm{m}$; the effective mass $m=0.05 m_{e}$; the relaxation time is $1 \mathrm{ps}$; and the Fermi energy $\epsilon_{F}=20 \mathrm{meV}$.

dicular spin accumulation is maximum at the edges of the sample, and the perpendicular spin accumulation at the edges of the sample will increase with the increase of the width of the sample. When the width $w$ of the sample is much larger than the spin diffusion length $D$, the perpendicular spin accumulation at the edges of the sample will approach a maximum value of $(e / 4 \pi) E_{x}\left(m \tau_{F} / \tau_{F}^{\uparrow} \epsilon_{F}\right)^{1 / 2}\left(1+\alpha^{2} m / \hbar^{2} \epsilon_{F}\right)$, which is independent of the sample width $w$. This will be a merit for the experimental measurement of the effect. In order to get a quantitative estimation of the perpendicular spin accumulation, let us consider some actual experimental parameters. In current 2DEG high quality samples, ${ }^{15,17,26}$ the typical carrier concentrations range from $5 \times 10^{11}$ to $10^{12} \mathrm{~cm}^{-2}$, the strength of the Rashba spin-orbit coupling is on the order of $1 \times 10^{-11}-5 \times 10^{-11} \mathrm{eV} \mathrm{m}$, the effective mass of conduction electrons is about $0.05 m_{e}$, the relaxation time is typically $1 \mathrm{ps}$, the spin diffusion length is about $1 \mu \mathrm{m}$, and the Fermi energy $\epsilon_{F}$ is about $20-50 \mathrm{meV}$. If one considers a sample with the width $w=10 \mu \mathrm{m}$ (much larger than the spin diffusion length) and the external field $e E_{x}=10 \mathrm{KeV} / \mathrm{m}$, then from Eq. (38) one can estimate that the perpendicular spin accumulation at the edges of the sample can be as large as $10^{-23} \mathrm{~J} \cdot \mathrm{s} / \mathrm{m}^{2}$. This magnitude should be large enough to be detected experimentally.

In conclusion, in this paper we have investigated the influences of spin accumulation on the intrinsic spin Hall effect in 2DEGs with Rashba spin-orbit coupling. We have presented a detailed theoretical analysis on the interplay between the spin Hall current and spin accumulation in the intrinsic spin Hall effect in a 2DEG. We have shown that in the presence of spin accumulation, the spin Hall conductivity will not have a universal value. The spin Hall current and spin accumulation in narrow strips of 2DEGs with Rashba spin-orbit coupling was calculated explicitly. The results show that in order to calculate correctly the spin Hall current and the spin Hall conductivity in a real sample with boundaries, the influences of spin accumulation need to be taken into account. Recently, Rashba pointed out that the Hamiltonian (1) implies that there exist nonvanishing dissipationless spin currents even in the thermodynamic equilibrium state (i.e., in the absence of the external electric field ). ${ }^{27}$ These background spin currents are not associated with real spin transports but spurious effects caused by the lacking of the time-reversal symmetry implied in the Hamiltonian (1). Due to this fact, a procedure for eliminating the spurious effects of these background spin currents should be devised in calculating transport spin currents if the background currents contribute to the calculation. But for the intrinsic spin Hall effect discussed in the present paper, the background spin currents do not contribute to the calculation of the spin Hall current due to the following reasons. First, the spin Hall current is polarized in the direction perpendicular to the $2 \mathrm{D}$ plane, while the background spin currents are polarized in the 2D plane. Second, the spin Hall current is a dynamic response of the spins to the external electric field and will vanish in the absence of the electric field, but the background spin currents are independent of the electric field. Due to these reasons, the background spin currents do not present in the calculation of the spin Hall current and hence do not need to be considered in the present paper.

This work is supported by the Research Grant Council of Hong Kong under Contract No. HKU 7088/01P and a CRCG grant from the University of Hong Kong (SQS), National Natural Science Foundation of China and 973 Project with No. 2002CB613504 (XHM and RBT), and National Science Foundation of China with No. 10474022 (LBH).
${ }^{1}$ G. A. Prinz, Science 282, 1660 (1998).

${ }^{2}$ S. A. Wolf, D. D. Awschalom, R. A. Buhrman, J. M. Daughton, S. von Molna, M. L. Roukes, A. Y. Chtchelkanova, and D. M. Treger, Science 294, 1488 (2001).

${ }^{3}$ P. Ball, Nature (London) 404, 918 (2000).

${ }^{4}$ M. Baibich, J. M. Broto, A. Fert, F. Nguyen Van Dau, F. Petroff, P. Etienna, G. Creuzet, A. Friederich, and J. Chazelas, Phys. Rev. Lett. 61, 2472 (1988).
${ }^{5}$ J. S. Moodera, L. R. Kinder, T. M. Wong, and R. Meservey, Phys. Rev. Lett. 74, 3273 (1995).

${ }^{6}$ M. Johnson and R. H. Silsbee, Phys. Rev. Lett. 55, 1790 (1985).

${ }^{7}$ M. Johnson, Phys. Rev. Lett. 70, 2142 (1993).

${ }^{8}$ T. Valet and A. Fert, Phys. Rev. B 48, 7099 (1993).

${ }^{9}$ P. R. Hammar, B. R. Bennett, M. J. Yang, and M. Johnson, Phys. Rev. Lett. 83, 203 (1999).

${ }^{10}$ G. Schmidt, D. Ferrand, L. W. Molenkamp, A. T. Filip, and B. J. 
van Wees, Phys. Rev. B 62, R4790 (2000).

${ }^{11}$ Y. Ohno, D. K. Young, B. Beschoten, F. Matsukura, H. Ohno, and D. D. Awschalom, Nature (London) 402, 790 (1999).

${ }^{12}$ R. Fiederling, M. Keim, G. Reuscher, W. Ossau, G. Schmidt, A. Waag, and L. W. Molenkamp, Nature (London) 402, 787 (1999).

${ }^{13}$ R. Mattana, J.-M. George, H. Jaffrès, F. van Dau, A. Fert, B. Lépine, A. Guivarc'h, and G. Jézéquel, Phys. Rev. Lett. 90, 166601 (2003).

${ }^{14}$ S. Murakami, N. Nagaosa, and S. C. Zhang, Science 301, 1348 (2003).

${ }^{15}$ J. Sinova, D. Culer, Q. Niu, N. A. Sinitsyn, T. Jungwirth, and A. H. MacDonald, Phys. Rev. Lett. 92, 126603 (2004).

${ }^{16}$ L. B. Hu, J. Gao, and S.-Q. Shen, cond-mat/0401231, 2004.

${ }^{17}$ D. Culcer, J. Sinova, N. A. Sinitsyn, T. Jungwirth, A. H. MacDonald, and Q. Niu, Phys. Rev. Lett. 93, 046602 (2004); J. Schliemann and D. Loss, Phys. Rev. B 69, 165315 (2004); J. P. $\mathrm{Hu}$, B. A. Bernevig, and C. Wu, Int. J. Mod. Phys. A 17, 5991 (2003); S. Q. Shen, Phys. Rev. B 70, 081311(R) (2004); N. A. Sinitsyn, E. M. Hankiewicz, W. Teizer, and J. Sinova, ibid. 70, 081312(R) (2004); S. Q. Shen, M. Ma, X. C. Xie, and F. C. Zhang, Phys. Rev. Lett. 92, 256603 (2004).

${ }^{18}$ L. Sheng, D. N. Sheng, and C. S. Ting, cond-mat/0409038, 2004.

${ }^{19}$ E. I. Rashba, Sov. Phys. Solid State 2, 1109 (1960); Y. A. Bychkov and E. I. Rashba, J. Phys. C 17, 6039 (1984).
${ }^{20}$ J. E. Hirsch, Phys. Rev. Lett. 83, 1834 (1999).

${ }^{21}$ S. Zhang, Phys. Rev. Lett. 85, 393 (2000).

${ }^{22}$ L. B. Hu, J. Gao, and S. Q. Shen, Phys. Rev. B 68, 153303 (2003); 68, 115302 (2003).

${ }^{23}$ Similar to Eq. (13), Eq. (27) is valid only if both spin-orbit split bands are occupied. In the presence of spin accumulation, the condition for both spin-orbit split bands are occupied is $\epsilon_{F}$ $-e \mu^{+}>-m \alpha^{2} / 2 \hbar^{2}$ (the bottom of the lower band) and $\epsilon_{F}$ $-e \mu^{-}>0$ (the bottom of the upper band). According to the relation of carrier density and Fermi level in 2DES, $n$ $=2 / \pi\left[\left(m \alpha / \hbar^{2}\right)^{2}+m \epsilon_{F} / \hbar^{2}\right]$ and we can get that the carrier density should be larger than a $\alpha$-dependent value $n>(2 / \pi)(\sqrt{3}$ $+5 / 2)\left(m \alpha / \hbar^{2}\right)^{2}$. For the parameters of 2DEG we use in the present paper the two conditions are fullfilled, and the two split bands are occupied. Usually, the band-dependent shifts $\mu^{+}$and $\mu^{-}$of the Fermi level are very small, so the occurrence of spin accumulation will not change the situation that whether the two spin-orbit split bands are both occupied or not.

${ }^{24}$ V. M. Edelstein, Solid State Commun. 73, 233 (1990).

${ }^{25}$ J. Inoue, G. E. W. Bauer, and L. W. Molenkamp, Phys. Rev. B 67, 033104 (2003).

${ }^{26}$ J. Nitta, T. Akazaki, and H. Takayanagi, Phys. Rev. Lett. 78, 1335 (1997).

${ }^{27}$ E. I. Rashba, Phys. Rev. B 68, 241315 (2003). 